\title{
Panorama teórico-analítico-sintético sobre a adoção de facetas no contexto da organização do conhecimento
}

\author{
Márcio Bezerra DA SILVA ${ }^{1[0000-0002-0052-7174]}$, \\ Zeny Duarte de MIRANDA2[0000-0003-0365-6905] \\ ${ }^{1}$ Faculdade de Ciência da Informação, Universidade de Brasília, Brasil. \\ marciobdsilva@unb.br \\ ${ }^{2}$ Instituto de Ciência da Informação, Universidade Federal da Bahia, Brasil. \\ zenyduegmail.com
}

\begin{abstract}
Resumen. Apresenta um panorama de uso de facetas na organização da informação, entre ambientes físicos e digitais. Fundamenta-se no contexto da organização do conhecimento, encarada como o arcabouço mais importante na teórica da organização da informação, e enfatiza-se na teoria da classificação facetada do indiano Ranganathan (1892-1972). Aborda três pontos básicos de discussão sobre a teoria ranganathiana: as cinco categorias fundamentais, personalidade-matéria-energia-espaço-tempo; a multidimensionalidade sistemática; e os cânones de renques e de cadeias de classes. Caracteriza-se como uma pesquisa exploratória, para identificar ambientes que usam facetas, e bibliográfica. Resulta que a maioria dos modelos de facetação está aplicado em ambientes digitais, que são modelos puramente acadêmico-profissionais, que os sistemas documentários (físicos) foram os suportes de aplicação mais adotados, que se identificaram modelos no período de 1933 até 2017 e que a categoria fundamental [Personalidade] foi a mais citada. Concluiu-se que a presença de facetas em sistemas de organização do conhecimento promulga que um mesmo produto tenha interpretações diferentes em uma mesma taxonomia, formaliza a multidimensionalidade idealizada na teoria da classificação facetada de Ranganathan, mas sem segui-la na íntegra por vezes, adota mnemônicas personalizadas e mostra que os ambientes onde são adotadas variam entre físicos e digitais, das bibliotecas físicas aos repositórios digitais.
\end{abstract}

Palabras clave: Organização da informação. Organização do conhecimento. Teoria da classificação facetada. Facetas. Categorias fundamentais.

Abstract. Presents an overview of the use of facets in the organization of in formation, between physical and digital environments. Is based on the context of the organization of knowledge, which is regarded as the most important framework in the theory of information organization, and is emphasized in Ranganathan's theory of faceted classification (1892-1972). Addresses three basic points of discussion about ranganathan theory: the five fundamental categories, personality-matter-energy-space-time; systematic multidimensionality; 
and the canons of ranks and class chains. Is characterized as an exploratory research, to identify environments that use facets, and bibliographical. The results are that most of the factional models are applied in digital environments, which are purely academic-professional models, that the documentary (physical) systems were the most adopted application supports, that models were identified in the period from 1933 to 2017 and that the fundamental category [Personality] was the most cited. Was concluded that the presence of facets in systems of knowledge organization promulgate that the same product have different interpretations in the same taxonomy, formalizes the multidimensionality idealized in Ranganathan's theory of the faceted classification, but without being followed in full in some cases, adopts custom mnemonics and shows that the environments where they are adopted vary from physical to digital, from physical libraries to digital repositories.

Keywords: Information organization. Knowledge organization. Theory of the faceted classification. Facets. Fundamental categories.

\section{Introdução}

Organizar pode ser considerado um ato intrínseco ao ser humano. Enquanto uma atividade que vem de longa data, a humanidade procura organizar o que se encontra em sua volta, entre pessoas, animais, objetos e fatos, segundo características específicas. Com o passar dos tempos, a ânsia por organizar o cotidiano passou a convergir, cada vez mais, à necessidade de tomar posse da informação que circunda o homem, ao enxergá-la como um insumo para a tomada de decisão. Neste sentido, caminha-se ao contexto da organização do conhecimento (OC), na qual encontram-se estudos que investigam a multidimensionalidade como um caminho para organizar a informação, inicialmente em ambientes físicos, mas que transcenderam aos digitais, ao acompanhar a dinamicidade imposta pelas mudanças na sociedade humana.

Pensar desta forma é ir ao encontro da teoria da classificação facetada (TCF), do indiano, matemático e bibliotecário Shiyali Ramamrita Ranganathan (1897-1972), ao trazer as cinco manifestações genéricas e distintas para classificar um recurso bibliográfico, comumente chamadas de facetas. De posse do objetivo de "[...] denotar qualquer componente de um domínio [...]" (GOMES; MOTTA; CAMPOS, 2006), as cinco manifestações, instruídas na obra intitulada Prolegomena to Library Classification, correspondem a mnemônica PMEST, traduzida em personalidade $[\mathrm{P}]$, matéria [M], energia [E], espaço [S] e tempo [T] (RANGANATHAN, 1960, 1963, 1967).

Apesar da compreensão de que a classificação facetada, enquanto uma proposta de categorização que ganhou notoriedade a partir da TCF do indiano Ranganathan, permite que um único assunto seja classificado em hierarquias diferentes a partir de múltiplos pontos de subjetividade, objetiva a estruturação do conhecimento, permite o mapeamento de uma área de assunto e inclui novos conceitos sem alterar a estrutura do sistema, dúvidas surgem sobre as formas de facetação criadas para a OI, das bibliotecas físicas à contemporaneidade dos ambientes digitais, tanto comerciais, quanto acadêmicos. 
Seguindo um percurso metodológico formalizado pelas técnicas de pesquisa exploratória e bibliográfica, e de coleta de dados em livros, artigos científicos, dissertações, teses e websites acadêmicos e comerciais, o presente artigo adota como objetivo geral o interesse em apresentar um panorama de uso de facetas na OI, entre ambientes físicos e digitais. Especificamente, verificar suportes físicos e digitais onde os modelos de facetação foram adotados, apresentar a evolução dos ambientes ao longo dos anos, identificar facetas à luz da mnemônica PMEST e elencar um exemplo de classificação facetada entre os modelos identificados.

\section{Teoria da Classificação Facetada: pontuações básicas}

A TCF baseia-se na ideia de two-fold infinity, ou seja, em duas visões, sendo uma pensada no leitor, correspondente as coleções de documentos, e outra pela ótica do universo de ideias a serem organizadas com base nas características dos documentos. $\mathrm{Na}$ verdade, Ranganathan defende à multiplicidade de visões de mundo e de necessidades informacionais, além de compreender que a natureza do universo do conhecimento é dinâmica. Esta dualidade permitirá características de flexibilidade e de capacidade de resposta, que inclusive poderá ser usada como medida de sucesso de qualquer sistema (RANGANATHAN, 1967).

Quantos aos postulados, também chamados de categoriais fundamentais (PMEST), referem-se a um termo genérico usado para denotar algum item, como um assunto básico $^{1}$ ou um isolado ${ }^{2}$, além de formar renques, termos e números (RANGANATHAN, 1967). Ainda segundo Ranganathan, "cada faceta de qualquer assunto, bem como cada foco nela, é reagida como uma manifestação de uma das cinco Categorias Fundamentais [...]" (RANGANATHAN, 1963, p. $1 * 25$, grifo do autor).

A CC, enquanto o primeiro sistema desenvolvido na perspectiva dinâmica, deflagrando a facetação como um novo tipo de classificação bibliográfica (CAMPOS, 2001), adota princípios para a confecção de sistemas multidimensionais e organizar a informação. Neste caso, ao tomar como base o plano das ideias (conceitos, mensagens), pois o cerne discursivo do artigo está no nível conceitual, elencam-se os cânones que afetam diretamente os arranjos taxonômicos, referentes a ordenação dos conceitos e as relações entre si. Entre eles, Gomes, Motta e Campos (2006) destacam os cânones de renques de classes e de cadeias de classes.

A formação dos renques (arrays), também denominados de ciclos (rounds), tratase de classes derivadas de um universo que possui uma única característica de divisão e assim estabelece-se uma ordenação na sequência preferida (RANGANATHAN, 1967). Os renques (figura 1) são arranjos em "[...] séries horizontais. Por exemplo:

${ }^{1}$ É um elemento sistematizado de ideias correspondentes a um campo (RANGANATHAN, 1967), ou seja, são "[...] áreas mais abrangentes do conhecimento, como Matemática, Agricultura" (CAMPOS, 2001, p. 49).

${ }^{2}$ É um elemento adotado para formar um componente de um assunto, mas que não pode ser considerado um assunto em si. Campos (2001) cita a ideia isolada Milho, que ao ser combinado com o assunto básico Agricultura define-se o assunto Agricultura do Milho. 
Macieira e Parreira são elementos da Classe Árvore Frutífera, formada pela característica de divisão - tipo de árvores frutíferas" (CAMPOS, 2001, p. 51).

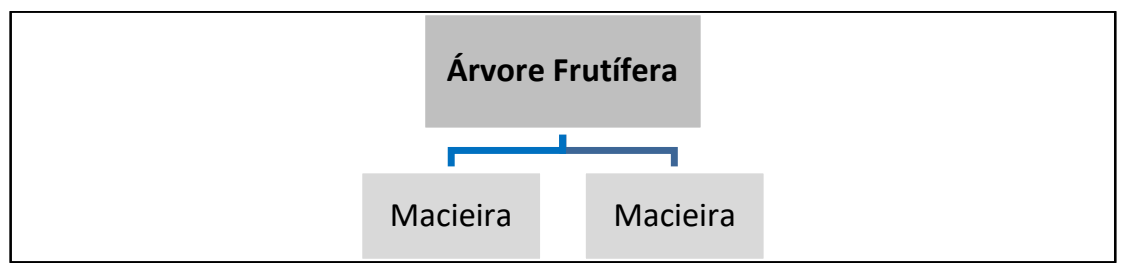

Figura 1. Renque, série horizontal.

A formação das cadeias (chain), do mesmo modo chamadas de níveis (levels), seria uma sequência formada por classes em um universo sequencial (1, 2, 3 e assim sucessivamente) até um ponto desejado (RANGANATHAN, 1967). Estruturando um arranjo em cadeias (figura 2) formam-se "[...] séries verticais de conceitos [...] conforme a cadeia descendente ou ascendente. Por exemplo: Macieira é um tipo de Árvore Frutífera, que, por sua vez, é um tipo de Árvore. Neste exemplo, observa-se uma cadeia descendente" (CAMPOS, 2001, p. 51).

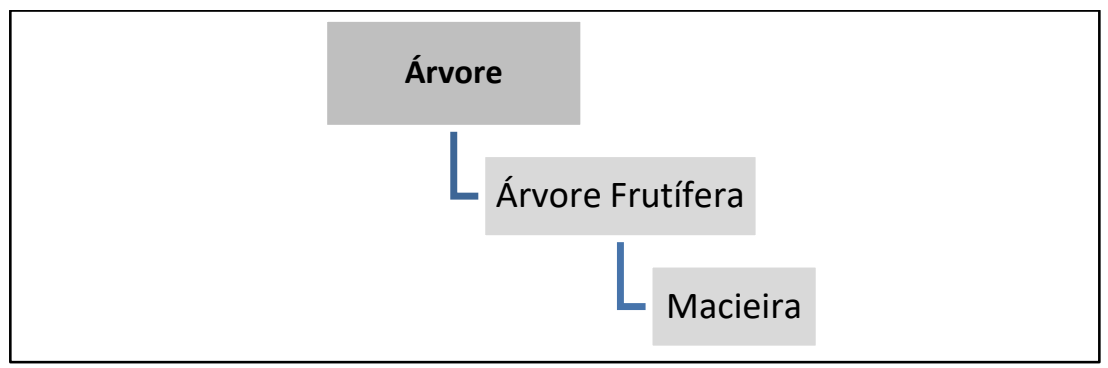

Figura 2. Cadeia, série horizontal.

Quanto mais horizontal for o sentido da taxonomia, maior será a exaustividade nas categorias fundamentais, e, por vezes, de facetas, apresentando características que estejam no mesmo nível de classificação. Por outro lado, quanto mais vertical for a estrutura da OI, mais profunda será o arranjo de conceitos, ou seja, uma especificidade mais acentuada, no sentido do mais amplo (top down) ao mais específico (bottom up).

\section{$3 \quad$ Resultados da pesquisa}

Em um caminho iniciado na organização de acervos físicos e que chegou ao mundo digital, as facetas se tornaram um viés aos sistemas de organização do conhecimento (SOC) multidimensionais, reconhecidos em sistemas documentários e tesauros, ambos em suporte físico, assim como em banco de dados (BD), software, biblioteca 
digital, lista eletrônica e tesauro digital, gerando a totalidade de 24 modelos de facetação identificados.

Encarados como esquemas que permitem a organização, o gerenciamento e a recuperação de informações (VICKERY, 2008), os SOC têm o seu núcleo de funcionamento lógico-relacional na classificação, vista por Ranganathan (1967, p. 395) como uma tarefa que busca "[...] mapear o universo multidimensional dos assuntos [...]". Ranganathan enxergava a classificação como uma ação de versatilidade sistêmica e que deveria acompanhar a evolução do conhecimento ao respeitar a dinamicidade e infinitude entre os conceitos, pensamento este que ia de encontro aos esquemas bibliográficos desenvolvidos na década de 1930 diante de suas dificuldades quanto a hospitalidade de novos assuntos no sistema.

A ideia de construir um sistema versátil está representada por 24 (100\%) modelos de facetação identificadas na literatura e que se concentram em maior número na perspectiva dos ambientes digitais segundo o quantitativo de 54\% (13) dos modelos, enquanto que $46 \%$ (11) respondeu aos espaços físicos.

Especificando os suportes de aplicação nos 24 modelos de facetação, sete suportes de aplicação foram identificados, sendo cinco de maneira digital e dois no viés físico. Apesar da maior quantidade de ambientes estar direcionada ao âmbito digital, o maior resultado do gráfico um (1) redireciona-se à perspectiva física, pois 36\% (8) representa os suportes em sistemas documentários (físicos), realidade comum nas bibliotecas, enquanto que os menores valores corresponderam aos suportes software e lista eletrônica, quantificados por $4 \%$ (1) cada uma.

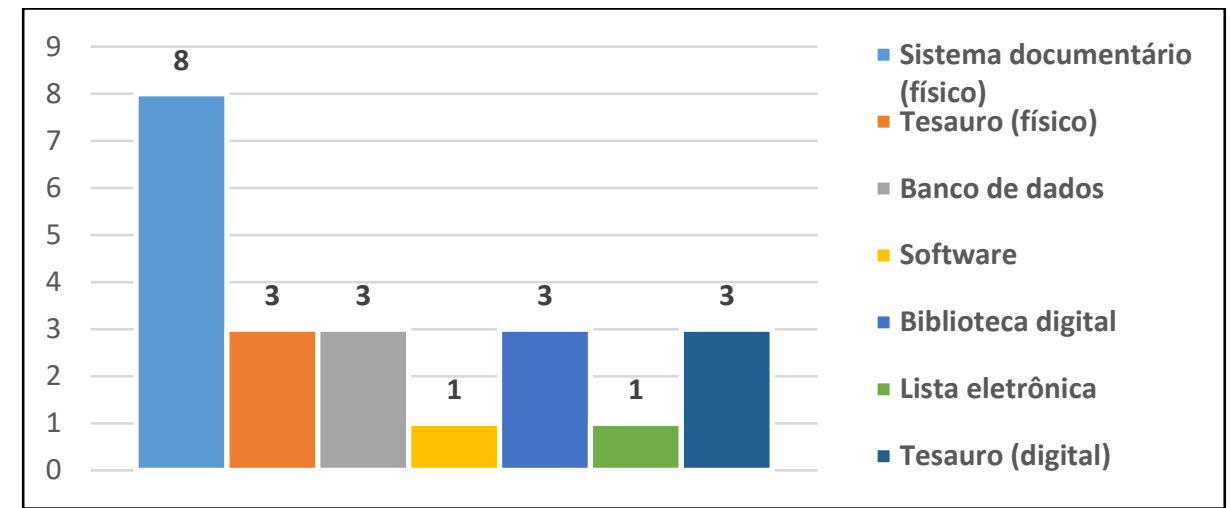

Gráfico 1. Suportes ${ }^{3}$ de aplicação ${ }^{4}$.

${ }^{3}$ Deve-se salientar que o BD Facet Analytical Theory - Humanities (FAT-HUM), desenvolvido pelo projeto intitulado Facet Analytical Theory in Knowledge Systems for Humanities (FATKS), foi quantificado apenas uma (1) vez, pois três (3) modelos de facetação foram usados no supracitado sistema eletrônico: duas na área de humanidades e uma em religião. Ou seja, foram identificados cinco BD, mas quantificados apenas três.

${ }^{4}$ Valores: $36 \%$ do total corresponde a um sistema documentário (físico); $14 \%$ a tesauro (físico), $\mathrm{BD}$, biblioteca digital e tesauro (digital); e $1 \%$ a software e lista eletrônica. 
Quanto ao seguimento evolutivo dos SOC facetados foi possível perceber que a linha se inicia em 1959 e mantêm-se até os tempos atuais, mostrando uma nítida continuidade dos estudos iniciados por Ranganathan na década de 1930, bem como marca a transcendência da aplicação desse tipo de sistema aos ambientes digitais (gráfico 2). Especificamente, os SOC facetados no âmbito digital iniciam-se em 2003, enquanto que os três últimos sistemas identificados no viés físico são de 2006, data que marca a transcendência dos modelos físicos para digitais. Contudo, todos os modelos de facetação correspondem ao escopo acadêmico-profissional, resultado que pode ser compreendido pela cognição de aproximação automática entre a $\mathrm{OC}$ e as bibliotecas (físicas e digitais), no sentido de organizar o conhecimento humano no universo da academia, entre livros, periódicos científicos, monografias, dissertações, teses etc.

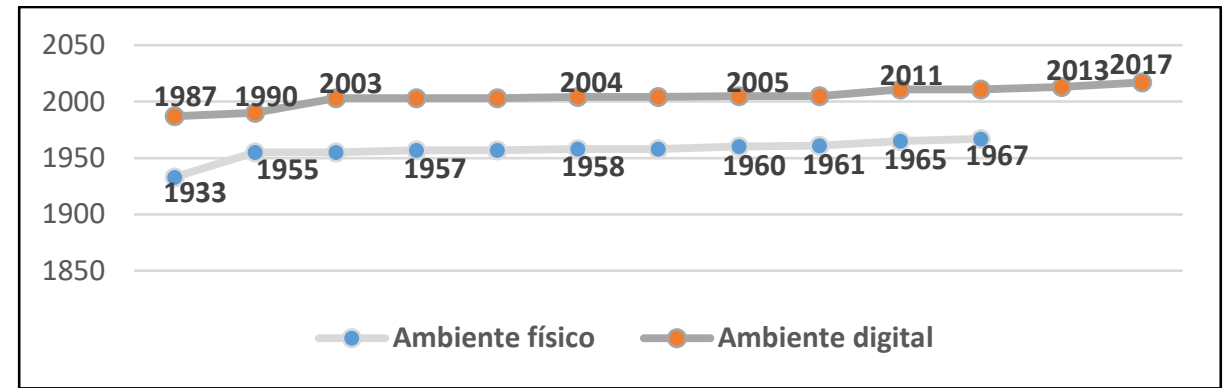

Gráfico 2. Evolução dos ambientes ao longo dos anos.

No que se referem as categorias fundamentais adotadas ao longo da breve evolução (gráfico 2), do ambiente físico ao digital, no total das 163 categorias identificadas e comparadas a mnemônica PMEST, a categoria [P] possui a maior quantidade de citações, correspondendo a 39\% (64), enquanto que o menor valor satisfez a categoria [T], valorada em 7\% (11) do total (gráfico 3). Além disso, o resultado por ser explicado tanto pelo conceito de cada categoria na mnemônica ou pela sequência (útil) de apresentação, ou seja, a ordem PMEST.

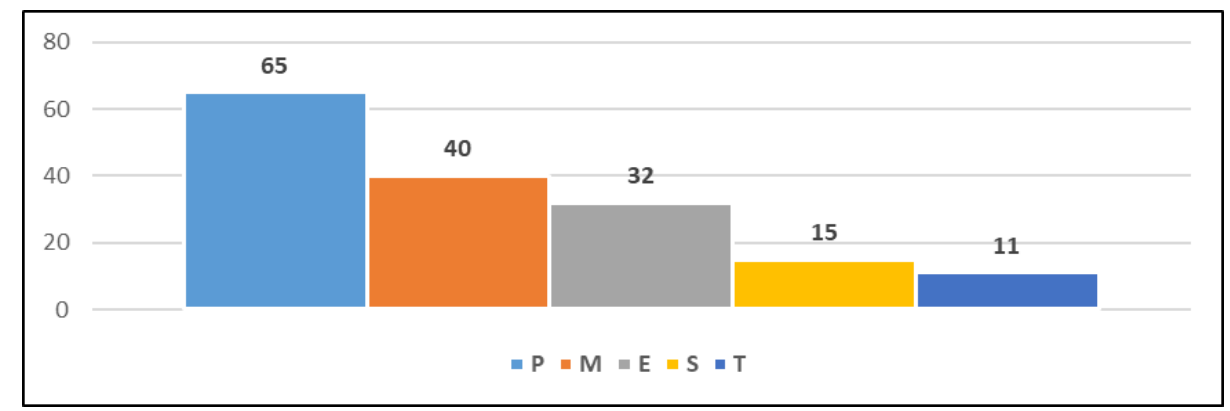

Gráfico 3. Categorias Fundamentais à luz do PMEST 5 .

${ }^{5}$ Valores: $39 \%$ do total corresponde a [P], 25\% a [M], 20\% a [E], 9\% a [S] e 7\% a [T]. 
Os resultados mostraram que a quantidade preponderante de modelos de facetação foi aplicada em ambientes digitais; que os sistemas documentários (físicos) foram os suportes de aplicação mais adotados; que a realização de modelos facetados no contexto da OC iniciou-se em 1933 e mantêm-se até os tempos atuais, sendo que os modelos digitais se iniciaram em 1987, data em que marca a transposição definitiva do físico ao digital, conforme identificações na literatura; que a categoria $[\mathrm{P}]$ foi a mais presente na mnemônica PMEST; e a adoção das categorias fundamentais estão em consonância com a sequência útil.

Adotando como exemplo um protótipo de BD (facetado), a sequência útil, como uma espécie de adaptação da utilizada na TFC, está representada pela tipologia [P]; matéria [M], como propriedade (qualidade) e matéria (substância); ação [E]; lugar [S] e tempo [T] (figura 3) (DA SILVA; NEVES, 2016). No protótipo, na classificação do livro "Introdução a Microinformática", foi gerada a sequência facetada "[P],[M],[M2],[T]", apresentando um segundo nível de renques entre "[M],[M2]" (matéria-propriedade). Alterando $[\mathrm{T}]$ por um novo $[\mathrm{P}]$, gerou-se a sequência “[P],[M],[M2],[2P]", isto é, um segundo clico de cadeias entre " $[\mathrm{P}],[2 \mathrm{P}]$ ”.

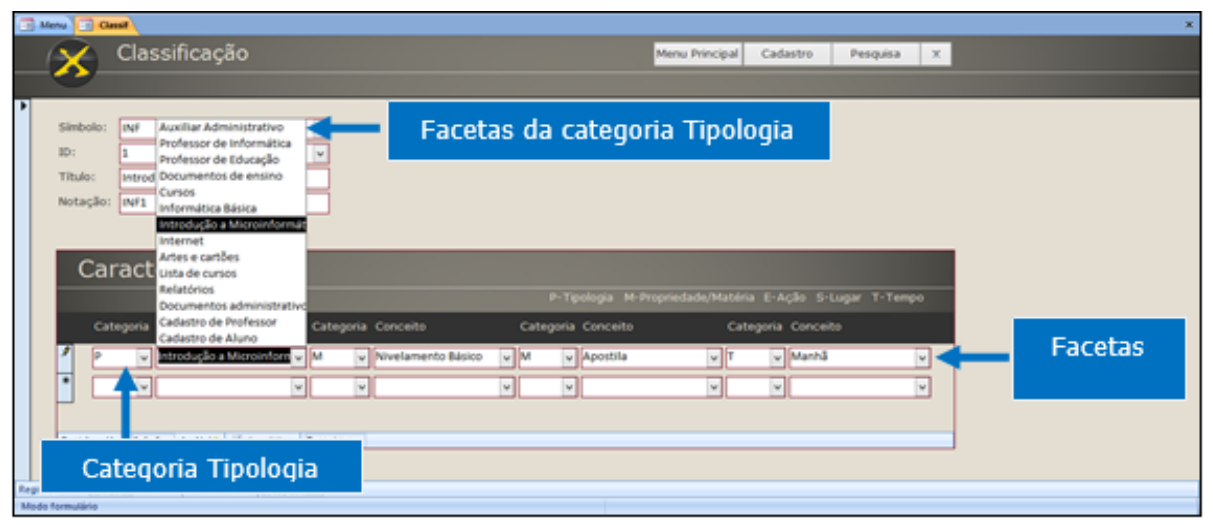

Figura 2. Exemplo de classificação facetada.

Fazendo a leitura (básica) da sequência “[P],[M],[M2],[T], para classificar a supracitada obra passou-se pela primeira rodada da personalidade $[\mathrm{P}]$, primeira rodada da matéria [M], segunda rodada da matéria [M2] e primeira rodada do tempo [T]. Quanto a sequência “[P],[M],[M2],[2P]", passou-se pela primeira rodada da personalidade $[\mathrm{P}]$, primeira rodada da matéria $[\mathrm{M}]$, segunda rodada da matéria [M2] e segundo nível da personalidade [2P].

\section{Considerações finais}

O presente trabalho apresentou um panorama onde destaca a importância da OI para a recuperação da informação, independentemente de o ambiente ser físico ou digital. Para tanto, o cerne aqui problematizado se deu na atividade da classificação, especialmente nos sistemas desenvolvidos com base em facetas. Trata-se de um anseio 
que encontra subsídios discursivos no campo da CI e desenvolvido em pesquisas realizadas no âmbito da OC. Neste sentido, em uma literatura identificada no contexto da OC foi possível identificar que a maioria dos modelos de facetação está aplicado em ambientes digitais, que são modelos puramente acadêmico-profissionais e não comerciais, que os sistemas documentários (físicos) foram os suportes de aplicação mais adotados, que foram identificados modelos no período de 1933 (TCF de Ranganathan) até 2017 e a categoria fundamental [P] foi a mais citada entre as dimensões do PMEST.

Concluiu-se que a presença de facetas em SOC promulga que um mesmo produto tenha interpretações diferentes em uma taxonomia navegacional, formalizam a multidimensionalidade idealizada na TCF de Ranganathan, mas sem segui-la na íntegra por vezes, adotam mnemônicas personalizadas e mostram que os websites assumiram a facetação como um meio que não se limita a organizar a informação, mas que influencia nas formas de navegação e busca de informação nas interfaces de ambientes digitais.

\section{Referencias}

CAMPOS, M. L. A. (2001). Linguagem documentária: teorias que fundamentam sua elaboração. Niterói: Ed. da UFF.

DA SILVA; M. B; BRITO, D. N. (2016). Em Revista.Ibero-Americana de Ciência da Informação, 9(1), 242-257. Recuperado em 12 de janeiro, 2019, de http://periodicos.unb.br/index.php/RICI/article/view/2236/1984.

GOMES, H. E.; MOTTA, D. F.; CAMPOS, M. L. de A. (2006). Revisitando Ranganathan: a classificação na rede. Biblioteconomia, Informação \& Tecnologia da Informação (BITI). Recuperado em 02 de janeiro, 2019, de http://www.conexaorio.com/biti/revisitando/revisitando.htm\#canones.

MILLS, J. (2004). Faceted classification and logical division in information retrieval. Library Trends 52(3) Winter, 541-570, Recuperado em 02 de janeiro, 2019, de https://www.ideals.illinois.edu/bitstream/handle/2142/1687/Mills541570.pdf?sequence=2\& isAllowed $=\mathrm{y}$.

RANGANATHAN, S. R. Colon Classification. Bombay: Asia Publishing House, 1963.

RANGANATHAN, S. R. Prolegomena to library classification. Bombay: Asia Publishing House, 1967.

RANGANATHAN, S. R. Reference service. Bombay: Asia Publishing House, 1961.

SATIJA, M. P. (2017). Colon Classification (CC). Encyclopedia of Knowledge Organization. Recuperado em 15 de janeiro, 2019, de http://www.isko.org/cyclo/colon_classification.

VICKERY, B. C. (2008). On 'knowledge organisation'. Recuperado em 02 de janeiro, 2019, de http://web.archive.org/web/20080404103206/www.lucis.me.uk/knowlorg.htm. 